\title{
The Guangzhou Twin Project
}

\author{
Mingguang He, ${ }^{1,2}$ Jian Ge,' Yingfeng Zheng,' Wenyong Huang,' and Junwen Zeng' \\ ' State Key Laboratory of Ophthalmology, Zhongshan Ophthalmic Center, Sun Yat-sen University, Guangzhou, China \\ ${ }^{2}$ School of Public Health, Sun Yat-sen University, Guangzhou, China
}

\begin{abstract}
The Guangzhou Twin Registry, initiated in 2005, is a population-based registry of twins born between 1987 and 2000. To date, over 9700 pairs of twins, regardless of their health and medical history, were enrolled in the database using the Official Household Registry of Guangzhou City. The twins were subsequently verified by door-to-door visits based on the registry address. The primary goal of this registry is to develop a resource for genetic epidemiological studies on common diseases in the southern Chinese population. The initial focus is to distinguish the genetic and environmental determinants of eye diseases, in particular myopia and glaucoma. About 1000 pairs of twins living close to the Zhongshan Ophthalmic Center were invited for the first phenotyping examination, questionnaire administration and DNA collection in July and August 2006. An annual eye examination and other phenotype data collection have been scheduled for up to 5 years in order to investigate changes in phenotypes including the myopia progression, physical development and the changes of other eye-related phenotypes. Recruitment of adult twins aged 50 years and over is underway in the same city with the assistance of the government.
\end{abstract}

\section{City Profile of Guangzhou: The Target Area for the Twin Registry}

Guangzhou is the capital city of Guangdong Province, and the center of its political, economic, scientific, educational and cultural life in south China. The city is located in the south of Guangdong Province, north of the Pearl River Delta. It lies close to the South China Sea, Hong Kong, and Macau. The city covers an area of 7434.4 square kilometers (2870 square miles) and is home to nearly 10 million people with permanent residency and a 3.7 million transitory population (Guangzhou Bureau of Statistics, 2003). Based on the multiple birth rates of about $1 \%$, we estimate the number of resident twins of all ages in the city should be over 100,000 twin pairs. The density and the large population size in this metropolitan city offer a unique opportunity to recruit a large number of twins and collect the phenotype and DNA data of the twins in a relatively short period of time and on a multiple visit basis.

\section{Twin Studies on Myopia: A Brief Overview of the Literature}

In recent years, researchers have conducted twin studies to evaluate the genetic mechanism of myopia. For example, the UK Twin Eye Study led by Hammond (Hammond et al., 2001) treated spherical equivalent as a quantitative trait and found a high heritability value (approximately $85 \%$ ) for the trait. Furthermore, using a subsample of dizygotic (DZ) twins who participated in the UK Twin Eye Study, Hammond et al. (2004) found linkage evidence on chromosomes 11p13, 3q26, 8p23, and 4q12.

From a study of younger twins in Denmark (Lyhne et al., 2001), Lyhne studied relatively young twins (aged 20-45), and regarded educational length (sum of education years) as a surrogate index for the environmental factor (close-up reading intensity). The heritability found in this study is similar to that of Hammond suggesting the high heritability of refractive error and its related biometry but not the environmental changes.

Unfortunately, although various twin studies of myopia supported the genetic contribution to the variation of myopia, these studies were hampered by technical limitations. For instance, twins in the United Kingdom study were mostly over 50 years old; the study also lacked cycloplegic refraction and ocular biometrics data. These limitations raise the nettlesome issue that presbyopia and nucleus sclerosis may have confounded the consonance of the phenotype. In addition, the studies were not able to include a sufficient number of twins affected by myopia as the rate of myopia is very low in European populations. Subsequent association analyses of Pax-6 under the peak of linkage evidence region on the chromosome 11 did not confirm the association.

Chinese are found to have the highest prevalence rate of myopia. On the basis of a recent survey of urban Chinese living in Guangzhou and the series of refractive error of school-aged children (RESC) studies, He et al. (2004) found the prevalence rate of

Received 3 August, 2006; accepted 8 September, 2006.

Address for correspondence: Mingguang He, Department of Preventive Ophthalmology, Zhongshan Ophthalmic Center, Guangzhou 510060, People's Republic of China. E-mail: mingguang_he@yahoo.com 
myopia in Chinese children four times as high as that in African and Hispanic children. The prevalence of myopia was shown to be as high as $78 \%$ in 15 -yearold children living in Guangzhou. However, it remains difficult to assess whether these ethnic variations are due to the difference in genetic susceptibility and/or environmental influence. The investigators in China and east Asia in general believe that environmental/ lifestyle differences, particularly the differences in schooling and close-up reading exposures, are likely to be responsible for the ethnic/racial variations in myopia (Morgan \& Rose, 2005). This theory appears to contradict the findings derived from family and twin studies in European populations. A twin study undertaken in Chinese with a more robust and careful study design should potentially be able to provide further insights on this issue.

\section{History of the Guangzhou Twin Project}

This ongoing twin registry in Guangzhou was initiated in 2005 and is maintained by research investigators $(\mathrm{MH})$ at the Zhongshan Ophthalmic Center (ZOC) of Zhongshan University, with initial focus on the genetic and environmental determinants of eye diseases, particularly myopia and glaucoma-related traits. The project was supported by a group of experienced ophthalmologists, optometrists and nurses. External advisors in the fields of genetics, twin research, medicine, epidemiology, ophthalmology and biostatistics provide external review and advice for the study. Funding for this twin project was limited at this stage. A small part of the funding was received from the Guangzhou City Scientific Fund while the majority of the funding and equipment were given by the Department of Preventive Ophthalmology in the ZOC hospital.

\section{Recruitment of Twins}

The twin database in Guangzhou now includes over 9000 pairs of twins born between 1987 and 2000. All twins were identified by the Resident Registry and then verified by both door-to-door visits and mailouts. The demographic characteristics of the identified twins were similar to the general population in Guangzhou as a whole (Table 1).

Listing in the Resident Registry is a legal requirement. It is kept by the police and details personal information for all permanent residents in the city. This database is updated on a yearly basis, collecting information on migration, death and new births. In early 2005, with the assistance of the Guangzhou City Bureau of Statistics (GCBS), those who shared the same birthday, home address, and household owner were selected from the Official Resident Registry as possible twins, given that twins less than 18 years old would have little chance of having different registered permanent residences. A population-based cohort born between 1987 and 2000 was selected, 20,626 individuals were identified as possible twins. After double-checking the address and parents' names, 1008 false twin individuals were dropped. Approximately 9709 pairs of twins were then enrolled, with an additional 64 triplets and 2 quadruplets. Detailed information in the database included the twin's surname, twin's first name, twin's birthday, household full address (including householder's name and his/her relationship to twins), parents' name and so forth.

Given that some contact information may be invalid or that some twin families do not live at the address recorded in the Resident Registry, door-todoor visits were undertaken by GCBS trained interviewers from January to March 2006. During this visit, interviewers first verified whether there were twin children living in the household at the given address. If there were twins, the interviewers verified the personal identity information of the twins, parents and head of the household. More detailed contact information including mobile phone and landline numbers were recorded in order to keep close contact and possibly send information by mobile phone SMS upon calling for phenotyping examination. A detailed questionnaire on zygosity, birth order, birthweight, gestational age, wearing of spectacles, parents' education and family socioeconomic status was also filled out during the door-to-door interview. At the end of the interview, staff gave all families a flyer notifying the on-going phenotyping examination July to August, 2006. Based on the availability of financial resources, time and the need for on-going phenotyping examination, these door-to-door visits were only conducted for twins aged 7 to 15 years (by the year of 2005) living in two districts close to the main eye hospital where the phenotypic examination would be undertaken. The twins aged 5 to 6 years and those aged 16 to 18 years, or those between 7 and 15 years but living in other districts in the city, were contacted by post at first and subsequent door-to-door verification for those who did not respond.

The door-to-door visits enabled access to 705 families from 1483 addresses. Of the remaining 778 families, 139 addresses were unable to be located, 270 occupied the address (confirmed by the neighbors) but were uncontactable therefore requiring second visits, 267 had moved from the address, and 102 families refused to participate. Of the 705 twin families successfully visited, 261 male same-sex twin pairs, 293 female same-sex twin pairs, 146 oppositesex twin pairs and 5 triplets were identified. In addition, $94.3 \%$ of twins had grown up and lived together since birth and $43.5 \%$ reported myopia and glass wearing in at least one twin member. The average birthweights were $2.5 \mathrm{~kg}(S D=0.5)$ for the older one and $2.4 \mathrm{~kg}(S D=0.5)$ for the younger one.

\section{Current Study}

The Guangzhou twin eye study is currently the major research focus. There are several overall aims of the study: estimating heritability and risk factors for myopia and glaucoma-related traits in young twins, the 
Table 1

Number of Twins and Other Multiples in the Guangzhou Twin Registry

\begin{tabular}{|c|c|c|c|c|c|c|}
\hline \multirow[t]{2}{*}{ Birth cohort (years) } & \multicolumn{3}{|c|}{ Twin pairs $N(\%)$} & \multirow[t]{2}{*}{ Twins subtotal } & \multirow[t]{2}{*}{ Triplet } & \multirow[t]{2}{*}{ Quadruplet } \\
\hline & Male-male & Female-female & Opposite-sex & & & \\
\hline 1987 & $272(38.4)$ & $254(35.8)$ & $183(25.8)$ & 709 & 1 & \\
\hline 1988 & $254(40.0)$ & $274(38.8)$ & $178(25.2)$ & 706 & 4 & \\
\hline 1989 & $266(38.4)$ & $253(36.5)$ & $174(25.1)$ & 693 & 5 & \\
\hline 1990 & $267(36.6)$ & $278(38.1)$ & $184(25.2)$ & 729 & 1 & \\
\hline 1991 & $242(40.0)$ & $229(36.0)$ & $166(26.1)$ & 637 & 7 & \\
\hline 1992 & $267(36.2)$ & $261(35.4)$ & $210(28.5)$ & 738 & 4 & \\
\hline 1993 & $286(38.8)$ & $272(35.0)$ & $219(28.2)$ & 777 & 2 & \\
\hline 1994 & $262(36.8)$ & $248(33.6)$ & $229(31.0)$ & 739 & 6 & \\
\hline 1995 & $277(37.7)$ & $259(35.3)$ & $198(27.0)$ & 734 & 6 & \\
\hline 1996 & $289(38.0)$ & $242(31.8)$ & $230(30.2)$ & 761 & 8 & 1 \\
\hline 1997 & $213(33.3)$ & $238(37.3)$ & $188(29.4)$ & 639 & 7 & \\
\hline 1998 & $248(38.0)$ & $213(32.6)$ & $192(29.4)$ & 653 & 3 & \\
\hline 1999 & $243(39.6)$ & $222(36.2)$ & $149(24.3)$ & 614 & 7 & 1 \\
\hline 2000 & $228(39.3)$ & $204(35.2)$ & $148(25.5)$ & 580 & 3 & \\
\hline Total & $3614(37.2)$ & 3447 (35.5) & $2648(27.3)$ & 9709 & 64 & 2 \\
\hline
\end{tabular}

discovery of genes that are relevant for these traits, and the comparison of heritability/mapping results from similar studies conducted in Australian twins.

Specific objectives are as follows:

1. To survey a representative population-based sample of young twins (7-9 and 13-15 years old) in urban Guangzhou with sufficient power to give a quantitative estimate of the heritability of myopia.

2. To perform a comprehensive ophthalmic examination (major focuses on myopia and glaucomarelated quantitative traits: visual acuity, refraction, intraocular pressure, corneal thickness, optic disc tomography, ocular biometry) of at least $300 \mathrm{DZ}$ and 300 monozygotic (MZ) twins.

3. To perform a prospective twin study with 5 years of follow-up quantifying close-up work (reading/writing, watching television, playing video games, computer use and outdoor activities) by the use of diaries, estimate the risk factors associated with the incidence and progress of myopia, and detect the gene-environment interaction in the onset and progression of myopia.

4. To perform an extended twin study involving young twins and their parents in order to asses the genes and environment interaction in the familial aggregation and transmission of the traits concerned.

5. To conduct the genome-wide single nucleotide polymorphism (SNP) scan on all DZ twin pairs and identify the loci and genes responsible for the quantitative traits concerned.
6. To compare the heritability estimation and mapping results of myopia in southern China with studies conducted in other populations (Australia and England).

A twin eye clinic was established at the end of June in the ZOC hospital. This clinic was fully equipped for sophisticated eye examination. Around 1000 twin pairs aged 7 to 15 years living close to the ZOC main hospital were invited for examination. The examinations for both eye and other phenotypes are summarized in Table 2. The parents of the young twins were also invited for examination on quantitative traits of eyes, for example, anterior chamber depth, corneal thickness, intraocular pressure, cycloplegic refraction, and optic disc tomography. A questionnaire that included questions on close-up reading intensity, birth records, parents' lifestyle, education and socioeconomic status was also administered by interviewers. At the end of the examination, approximately $10 \mathrm{ml}$ of blood was taken from all young twins for zygosity testing and DNA extraction. Ethical approval of the study was obtained from the Zhongshan University Ethical Review Board and the Ethical Committee of ZOC. The study was conducted in accordance with the Tenets of the World Medical Association's declaration of Helsinki. Before any examination, a written informed consent was obtained from the parents or guardians of all young twins after a careful explanation of the project including risk and benefit of examination. Permission to use the DNA data was also obtained. In the first 3 weeks of the examination (up to the date of submitting this manuscript), a total of 275 pairs of twins and their parents were successfully examined. The data collection efforts were still ongoing. 
Table 2

The Phenotype Data Planned for Collection in the Twin Eye Study

\begin{tabular}{|c|c|}
\hline Phenotypes & Data collection method \\
\hline \multicolumn{2}{|l|}{ Eye-related phenotypes } \\
\hline Cycloplegic refraction (spherical equivalent) & Autorefractor refined by subjective refraction \\
\hline Keratometry (radius of cornea) & Autorefractor \\
\hline Anterior chamber depth & Optical pachymetry \\
\hline Corneal thickness & Optical pachymetry \\
\hline Intraocular pressure & Tonopen and noncontact tonometer \\
\hline Axial length & IOLMaster \\
\hline Stereoacuity & Randot Stereo Chart \\
\hline Color test & Standard color test chart \\
\hline Optic disc tomography & Heidelberg retinal tomography (HRT) III \\
\hline \multicolumn{2}{|l|}{ Other phenotypes } \\
\hline \multicolumn{2}{|l|}{$\begin{array}{l}\text { Weight, height, blood pressure, waist-hip ratio, } \\
\text { arm circumference, triceps skin fold thickness }\end{array}$} \\
\hline Blood cell count & Blood test \\
\hline
\end{tabular}

\section{Future Plans}

Further enhancement of the twin registry. To date, a website in Chinese has been established for communication between twin families and research investigators, and two newsletters have been sent to the twin families in order to report the progress of twin project. The newsletter will be sent on a bimonthly basis. Twin family gatherings are arranged every month and roughly 50 twin families are invited. Twin families were also asked to give the contact details of a close friend or relative. In so doing, the twins are now able to be contacted in the event that they change address without notifying us. Two more staff members will be hired to take care of the administrative and logistic work of this project.

Five-year follow-up of the young twins involved in the twin eye study. All the twins who were enrolled and completed baseline examination in July and August of 2006 have been invited for the annual examination again during the school summer holidays for the next 5 years. The project will give all children in need spectacles, free of charge, for 5 years. We hope the free spectacle provision will encourage the participation for this long-term prospective follow-up. As a result of this prospective program, we have the opportunity to examine the genetic and environmental determinants for the onset and the progression of myopia. Collaboration with investigators interested in phenotypes other than myopia is possible with the study, and these phenotypes could be collected during the 5-year follow-up. Such collaboration is strongly encouraged.

Expansion of the enrolment of the twins aged 50 years and over in the same city. Given that older individuals aged 50 years and over are more likely be affected by eye diseases and other general health problems, we plan to initiate the adult twin registry in the same city at the end of 2006. The adult twins will be identified through a governmental community administrative system. The City Department of Civil and Administration has agreed to assist the work through their Community Service Center administrative units distributed throughout communities in the city. The reputation built by the young twin project and growing experience of the staffs will hopefully facilitate further efforts in the enrolment of this adult twin cohort in Guangzhou.

In general, the twin eye study based on our established twin registry in Guangzhou is an opportunity to conduct for the first time an epidemiologically valid, technically detailed study on the genetic and environmental determinants of myopia in the Chinese, a population highly affected by myopia. A long-term perspective follow-up of this large twin cohort will offer unique resources for genetic research on the common complex diseases of the eye and others. Further expansion of the twin registry to include adult twins is a new opportunity for the investigation of common diseases affecting the elderly.

\section{Acknowledgments}

This work is supported in part by a grant from the Guangzhou Scientific Committee. Dr David Mackey and his team members from University of Melbourne, Dr Paul Foster from Moorfields Eye Hospital London, and Dr Christ Hammond from St Thomas Hospital London all provided invaluable technical advice during the preparation of the project. Mr James Console helped with the proofreading and preparation of this manuscript.

\section{References}

Guangzhou Bureau of Statistics. (2003). Main indicators of National Economic by district and county-level 
city. Retrieved August, 2006, from http://www.gzstats. gov.cn/Admin/sjcx/tjnj/2004/01/1-28.htm

Hammond, C. J., Andrew, T., Barber, M. J., \& Spector, T. D. (2004). A susceptibility locus for myopia in the normal population is linked to the PAX6 gene region on chromosome 11: A genomewide scan of dizygotic twins. American Journal of Human Genetics, 75, 294-304.

Hammond, C. J., Snieder, H., Gilbert, C. E., \& Spector, T. D. (2001). Genes and environment in refractive error: The twin eye study. Investigative Ophthalmology and Visual Science, 42, 1232-1236.
He, M., Zeng, J., Xu, J., Pokharel, G. P., \& Ellwein, L. B. (2004). Refractive error and visual impairment in urban children in southern China. Investigative Ophthalmology and Visual Science, 45, 793-799.

Lyhne, N., Sjolie, A. K., Kyvik, K. O., \& Green, A. (2001). The importance of genes and environment for ocular refraction and its determiners: A population based study among 20-45 year old twins. British Journal of Ophthalmology, 85, 1470-1476.

Morgan, I., \& Rose, K. (2005). How genetic is school myopia? Progress in Retinal and Eye Research, 24, $1-38$. 